\title{
Implementation of repeat biopsy and detection of cancer after a diagnosis of atypical small acinar proliferation of the prostate
}

\author{
TAKAHIRO IMANAKA, TAKAHIRO YOSHIDA, AYUMU TANIGUCHI, \\ KAZUAKI YAMANAKA, HIDEFUMI KISHIKAWA and KENJI NISHIMURA \\ Department of Urology, Hyogo Prefectural Nishinomiya Hospital, Nishinomiya, Hyōgo 662-0918, Japan
}

Received December 31, 2019; Accepted August 28, 2020

DOI: $10.3892 / \operatorname{mco} .2020 .2137$

\begin{abstract}
Current guidelines recommend a repeat biopsy within 3-6 months after an initial diagnosis of atypical small acinar proliferation (ASAP) due to the high incidence of cancer detection on repeat biopsy. The current study sought to investigate practice patterns after a diagnosis of ASAP in a real-world setting and examine the clinicopathological outcomes of repeat biopsy. The departmental database of the Hyogo Prefectural Nishinomiya Hospital identified 97 of 1,218 patients with a diagnosis of ASAP on initial biopsy from 2011 to 2016. Clinical and pathological data were retrospectively analyzed. Of the 97 patients, 34 (35.1\%) underwent a repeat biopsy. Patients with a repeat biopsy had a significantly higher prostate-specific antigen (PSA) velocity and shorter PSA doubling time than patients without a repeat biopsy $(\mathrm{P}=0.0002)$, and of these 34 patients with a repeat biopsy, $16(47.1 \%)$ were diagnosed as having cancer. Multivariate logistic regression analysis revealed that a small prostate $(\mathrm{P}=0.0250)$ and advanced age $(\mathrm{P}=0.0297)$ were associated with cancer detection on repeat biopsy. Of the 16 cancers identified, $13(81.6 \%)$ were diagnosed with a Gleason score $>6$. The results indicated that the implementation of a repeat biopsy for patients with ASAP could be affected by clinical characteristics in real-world settings, despite the current recommendation of guidelines endorsing immediate repeat biopsy. Prostate volume and age would aid in the decision-making process to perform repeat biopsy in patients with high PSA velocity and short PSA doubling time after a diagnosis of ASAP.
\end{abstract}

\section{Introduction}

Prostate cancer is a significant health concern worldwide. For instance, prostate cancer remains the most commonly

Correspondence to: Dr Takahiro Yoshida, Department of Urology, Hyogo Prefectural Nishinomiya Hospital, 13-9 Rokutanji, Nishinomiya, Hyōgo 662-0918, Japan

E-mail: yakinikugohan@gmail.com

Key words: prostate cancer, atypical small acinar proliferation, repeat biopsy, prostate volume, PSA velocity, PSA doubling time diagnosed solid organ tumor in the United States, with an estimated 161,360 new cases and 26,730 deaths in 2016 (1). The incidence of prostate cancer is also increasing in most Asian countries possibly due to growing implementation of prostate-specific antigen (PSA) testing (2), with the morbidity ranking 4th among Japanese men in 2014 (3). Prostate cancer comprises a wide spectrum of diseases with some tumors being indolent and manageable with a monitoring strategy and others being so aggressive that radical treatments are required to reduce the risk of death from the disease (4). Therefore, the ideal management of prostate cancer requires an individualized treatment strategy. As a definitive diagnosis of prostate cancer is usually made through needle biopsy of the prostate gland, precise differential diagnosis on biopsy specimens to distinguish indolent tumors from aggressive disease is a key to offering proper management for individual patients to avoid over- or undertreatment of prostate cancer (5).

Atypical small acinar proliferation (ASAP) is defined as a lesion without an adequate amount of histologic atypia to establish a definitive diagnosis of prostate adenocarcinoma (6). ASAP was reported to be found in 5-10\% of needle biopsies of prostate glands (7). Previous studies showed that on average, $40 \%$ of patients with ASAP on initial biopsy were found to harbor adenocarcinoma on repeat biopsy $(8,9)$. Accordingly, the current US and European guidelines recommend immediate repeat biopsy within 3-6 months after an initial diagnosis of ASAP $(10,11)$. However, the evidence on ASAP is still limited, and no prospective study on ASAP has been reported so far. Remarkably, the probability of cancer found on repeat biopsy ranges from 20.0 to $71.6 \%$ (12-22). Clinical and pathological predictors reported for cancer detection on repeat biopsy after an ASAP diagnosis vary among studies $(7,12,16,18,23,24)$, but these variations could result from the retrospective nature of the studies. Of note, most of the studies lacked information on factors that could influence patient selection for repeat biopsy after a diagnosis of ASAP on initial biopsy, which would be crucial to interpreting the results.

In general, recommendations from the guidelines are not fully implemented in daily practice, where a variety of factors, including physician's discretion and patient's preference, influence clinical decisions. In this study, we first sought to investigate practice patterns after a diagnosis of ASAP in a real-world setting, focusing on factors that could affect patient selection for repeat biopsy after the ASAP diagnosis. We also 
analyzed clinical factors associated with the detection of cancer on repeat biopsy after a diagnosis of ASAP and pathologically characterized the cancer detected on repeat biopsy.

\section{Materials and methods}

Patient population. We retrospectively searched the departmental database on pathological reports of prostate biopsy performed at the Hyogo Prefectural Nishinomiya Hospital from January 2011 to December 2016. From 1,218 initial prostate biopsies, we identified 97 patients with a diagnosis of ASAP without concomitant cancer. All 97 patients were enrolled in this study. Clinical and pathological information of the patients was collected from the electronic medical record. This study was approved by the local institutional research ethics board (H30-37).

Prostate biopsy. All patients underwent transperineal prostate biopsy under the guidance of transrectal ultrasonography. Until March 2013, we performed biopsy with a standard 10-core template, but the biopsy protocol was changed to a 12-core template in April 2014. Additional targeted biopsy was carried out when a suspicious lesion was pointed out by an institutional radiologist on pre-biopsy magnetic resonance imaging. The diagnosis of ASAP and cancer was confirmed by a single certified pathologist at our institution throughout the study period. The presence of basal cells was routinely assessed using $34 \beta E 12$ immunostaining. A repeat biopsy was performed based on the urologists' discretion and patients' preference.

PSA kinetics. PSA kinetics including velocity and doubling time were calculated using the web-based calculation tool of the Memorial Sloan Kettering Cancer Center (25). All PSA values measured from the time of initial biopsy to the time of repeat biopsy were used to calculate PSA kinetics for patients with repeat biopsy. Patients with a negative PSA doubling time were assigned a PSA doubling time of 100 months so as to be included for the linear analyses following preceding studies (26). For comparison, PSA values measured within one year after initial biopsy were used to calculate PSA kinetics for patients without repeat biopsy because the median time from initial biopsy to repeat biopsy in the patients with repeat biopsy was 354.5 days.

Statistical analysis. Continuous variables are presented as the median value and range. Continuous and categorical variables were compared using the Mann-Whitney U test and the Chi-square test, respectively. Multivariate analysis was conducted by logistic regression analysis. Variables were entered into multivariate analysis if a P-value was $<0.1$ according to the univariate analysis. A P-value $<0.05$ was considered statistically significant. All statistical analyses were performed using JMP version 11.0.0 (SAS Institute Inc.).

\section{Results}

Fig. 1 shows a diagrammatic representation of the clinical courses of the patients who were diagnosed as having ASAP on initial biopsy of the prostate. In total, 1,218 patients who received an initial biopsy were identified, and $97(8.0 \%)$ were diagnosed as having ASAP. Thirty-four (35.1\%) of these 97 patients

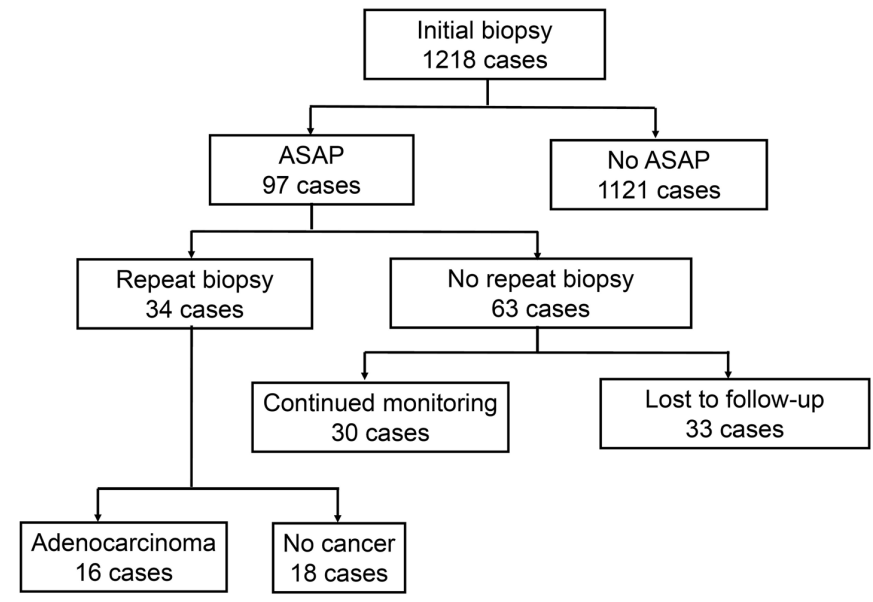

Figure 1. Diagrammatic representation of the clinical course of patients diagnosed with ASAP on initial biopsy of the prostate gland. ASAP, atypical small acinar proliferation.

underwent a repeat biopsy, whereas the remaining 63 did not. Thirty of these 63 patients were followed after an initial biopsy at our institution, whereas the remaining 33 were either lost to follow up or were introduced to a local clinic for follow-up. Of the 34 patients who received a repeat biopsy, 16 (47.1\%) were found to have prostate adenocarcinoma.

Table I provides clinical characteristics at the time of the repeat biopsy in the 34 patients receiving repeat biopsy. Because the median time to repeat biopsy in these patients was 354.5 days, clinical characteristics at one year after initial biopsy of the 30 patients without repeat biopsy were also summarized in Table I for comparison. Of the 34 and 30 patients with and without repeat biopsy, 3 and 14 had declining PSA values over time, respectively, and consequently had negative values of PSA velocity and PSA doubling time. These 17 patients were assigned a PSA doubling time of 100 months. Age, prostate volume, PSA value, and PSA density were not significantly different between the patients with and without repeat biopsy. Among the variables examined, PSA velocity and PSA doubling time were significantly different between the two groups (1.4 vs. $-0.9 \mathrm{ng} / \mathrm{ml} / \mathrm{year}$ and 29.3 vs. 100.0 months, $\mathrm{P}=0.0002$ and 0.0002 , respectively) (Table I).

Next, we compared clinical characteristics of the patients with and without cancer on repeat biopsy (Table II). Compared to the patients without cancer, those with cancer had a significantly smaller prostate volume ( 33.2 vs. $47.9 \mathrm{ml}, \mathrm{P}=0.0473$ ) and higher PSA density ( 0.28 vs. $0.17 \mathrm{ng} / \mathrm{ml} / \mathrm{ml}, \mathrm{P}=0.0324)$ on univariate analysis. Multivariate analysis subsequently identified increasing age (OR 1.20; 95\% CI, 1.02-1.50; $\mathrm{P}=0.0297)$ and small prostate volume (OR 0.92; 95\% CI, 0.86-0.99; $\mathrm{P}=0.0250)$ to be significantly associated with cancer detection on repeat biopsy (Table III).

Table IV summarizes the pathological features of the 16 cases in which cancer was detected on repeat biopsy. Of these 16 patients, 3 (18.7), 6 (37.5), 2 (12.5), and 5 (31.3\%) had Gleason scores of $3+3,3+4,4+3$, and $4+4$, respectively. The number of positive cores was 1, 2, and 3 in 7 (43.8), 3 (18.7), and $6(37.5 \%)$ patients, respectively. The maximal involvement of cancer in a positive core was $<50$ in $11(68.7)$ and $\geq 50 \%$ in $5(31.3 \%)$ patients, respectively. 
Table I. Demographic and clinicopathological characteristics of patients with and without repeat biopsy.

\begin{tabular}{|c|c|c|c|c|c|}
\hline \multirow{2}{*}{$\begin{array}{l}\text { Characteristic } \\
\text { Age, years median (range) }\end{array}$} & \multicolumn{2}{|c|}{ Repeat biopsy $(n=34)$} & \multicolumn{2}{|c|}{ No repeat biopsy $(n=30)$} & \multirow{2}{*}{$\begin{array}{r}\text { P-value } \\
0.1149\end{array}$} \\
\hline & 71.5 & $(56-82)$ & 76.0 & $(45-86)$ & \\
\hline PSA, ng/ml median (range) & 9.6 & $(1.7-30.2)$ & 7.7 & $(3.1-30.4)$ & 0.4432 \\
\hline Volume, ml median (range) & 38.0 & $(20.3-72.1)$ & 43.8 & $(22.0-85.0)$ & 0.3293 \\
\hline PSAD, ng/ml/ml median (range) & 0.21 & $(0.03-1.02)$ & 0.18 & $(0.05-0.50)$ & 0.2364 \\
\hline Time to repeat biopsy, days median (range) & 355 & $(19-1994)$ & - & & \\
\hline PSA velocity, ng/ml/year median (range) & 1.4 & $(-2.4-20.6)$ & -0.9 & $(-20.0-3.9)$ & 0.0002 \\
\hline PSA doubling time, months median (range) & 29.3 & $(6.4-105.5)$ & 100.0 & $(16.2-694.3)$ & 0.0002 \\
\hline
\end{tabular}

${ }^{a}$ Mann-Whitney U test. PSA, prostate-specific antigen; PSAD, PSA density.

Table II. Clinical factors of cohorts with and without cancer on repeat biopsy following a diagnosis of atypical small acinar proliferation.

\begin{tabular}{|c|c|c|c|c|c|}
\hline \multirow{2}{*}{$\begin{array}{l}\text { Factor } \\
\text { Age, years median (range) }\end{array}$} & \multicolumn{2}{|c|}{ Cancer $(n=16)$} & \multicolumn{2}{|c|}{ No cancer $(n=18)$} & \multirow{2}{*}{$\begin{array}{c}\text { P-value } \\
0.1359\end{array}$} \\
\hline & 73.0 & $(65-82)$ & 71.5 & $(56-76)$ & \\
\hline PSA, ng/ml median (range) & 9.2 & $(4.5-29)$ & 9.5 & $(1.7-30.2)$ & $0.6662^{\mathrm{a}}$ \\
\hline Volume, ml median (range) & 33.2 & $(20.3-64.7)$ & 47.9 & $(25.8-72.1)$ & $0.0473^{\mathrm{a}}$ \\
\hline PSAD, ng/ml/ml median (range) & 0.28 & $(0.11-0.99)$ & 0.17 & $(0.03-1.02)$ & $0.0324^{\mathrm{a}}$ \\
\hline Time to repeat biopsy, days median (range) & 413.5 & $(50-1994)$ & 269.5 & $(19-1231)$ & $0.1205^{\mathrm{a}}$ \\
\hline \multicolumn{6}{|l|}{ Type of biopsy } \\
\hline Systematic & 5 & & 11 & & $0.0817^{\mathrm{b}}$ \\
\hline Systematic + target & 11 & & 7 & & \\
\hline PSA velocity, ng/ml/year median (range) & 1.4 & $(-2.4-7.8)$ & 1.1 & $(-9.4-20.6)$ & $0.9781^{\mathrm{a}}$ \\
\hline PSA doubling time, months median (range) & 34.9 & $(13.9-105.5)$ & 37.2 & $(6.4-100.0)$ & $0.5000^{\mathrm{a}}$ \\
\hline
\end{tabular}

${ }^{\mathrm{a}}$ Mann-Whitney U test; ${ }^{\mathrm{b}} \mathrm{Chi}$-square test. PSA, prostate-specific antigen; PSAD, PSA density.

Table III. Multivariate logistic regression analysis for the identification of factors associated with cancer detected on repeat biopsy following a diagnosis of atypical small acinar proliferation.

\begin{tabular}{lccc}
\hline & Univariate & \multicolumn{2}{c}{ Multivariate } \\
\cline { 3 - 4 } Variable & P-value & OR & $(95 \%$ CI $)$ \\
\hline Age, years & 0.0741 & 1.20 & $(1.02-1.50)$ \\
Volume, $\mathrm{ml}$ & 0.0437 & 0.93 & $(0.86-0.99)$ \\
PSA, $\mathrm{ng} / \mathrm{ml}$ & 0.5244 & & 0.0297 \\
PSAD, $\mathrm{ng} / \mathrm{ml} / \mathrm{ml}$ & 0.2181 & 0.70 & $(0.01-105.01)$ \\
PSA velocity, ng/ml/year & 0.2500 & & 0.0657 \\
PSA doubling time, months & 0.6600 & & \\
\hline
\end{tabular}

OR, odds ratio; CI, confidence interval; PSA, prostate-specific antigen; PSAD, PSA density.

\section{Discussion}

In this study, we found that patients with higher PSA velocity and shorter PSA doubling time were more susceptible to repeat biopsy among patients who were found to have ASAP on initial biopsy and followed by urologists in a real-world practice. In this cohort, $47.1 \%$ were found to have prostate adenocarcinoma on repeat biopsy. Multivariate analysis found that small prostate volume and increasing age were significantly associated with cancer detection on repeat biopsy. Of the cancers found on repeat biopsy, $81.3 \%$ were diagnosed as Gleason score greater than 6 . 
Table IV. Pathological features of 16 cases in which cancer was detected on repeat biopsy following a diagnosis of atypical small acinar proliferation.

\begin{tabular}{lc}
\hline Variable & $\mathrm{n}(\%)$ \\
\hline $\begin{array}{l}\text { Gleason score } \\
3+3\end{array}$ & $3(18.7)$ \\
$3+4$ & $6(37.5)$ \\
$4+3$ & $2(12.5)$ \\
$4+4$ & $5(31.3)$ \\
Number of positive cores & \\
1 & $7(43.8)$ \\
2 & $3(18.7)$ \\
3 & $6(37.5)$ \\
PSAD, n $\%)$ & \\
$\quad \leq 0.15$ & $3(18.9)$ \\
$>0.15$ & $13(81.1)$ \\
Maximal involvement of cancer in a positive core & \\
$<50$ & $11(68.7)$ \\
$\geq 50$ & $5(31.3)$ \\
\hline
\end{tabular}

PSAD, prostate-specific antigen density.

Describing the characteristics of patients both with and without a repeat biopsy is important to proper interpretation of the analysis results of repeat biopsy because the implementation of a repeat biopsy should be biased in a retrospectively collected cohort. However, only a few studies have mentioned possible factors biasing patient selection for repeat biopsy after a diagnosis of ASAP $(12,14)$. One study reported that patients with a repeat biopsy were younger than those without a repeat biopsy (14), and the other reported that patients with a repeat biopsy had a higher Charlson Comorbidity Index score than patients without a repeat biopsy (12). In the present study, patients who received a repeat biopsy had higher PSA velocity and shorter PSA doubling time than those who did not. These data indicate that the implementation of a repeat biopsy for patients with ASAP could be affected by the patient's characteristics in a real-world setting despite the current recommendation of the guidelines endorsing immediate repeat biopsy within 3-6 months after an initial diagnosis of ASAP $(10,11)$. We need to take these biases into consideration when interpreting the results in retrospective studies on repeat biopsy after a diagnosis of ASAP.

In the current cohort, small prostate volume and increasing age were statistically significant predictors for cancer detection on repeat biopsy after a diagnosis of ASAP. This finding is consistent with those in some of the preceding studies $(16,18)$. In general, increasing age is a well-established risk factor for prostate cancer (11). As for prostate volume, the standard systemic biopsy with a fixed number of biopsy cores failed to detect cancer in large prostates $(24,27,28)$. This could be one reason, therefore, that small prostate volume was found to be a predictor for cancer detection in the present analysis. Actually, the rate of cancer detection was reported to rise as the number of biopsy cores was increased on repeat biopsy for large prostates in patients with a previous diagnosis of ASAP (21). The increasing number of cores would contribute to the detection of cancer on repeat biopsy after a diagnosis of ASAP, especially for patients with large prostates.

The probability of Gleason score greater than 6 , which is considered high-grade cancer, on repeat biopsy after a diagnosis of ASAP substantially varies from 8.0 to $66.7 \%$ among different studies $(12,14-17,19,21,22)$. In the present cohort, $13(81.3 \%)$ of the 16 cancers were diagnosed as Gleason score greater than 6 on repeat biopsy after a diagnosis of ASAP, which is higher than the rates in the preceding reports. The high incidence of Gleason score greater than 6 in the current cohort may partly be explained by interobserver variability (7). Nakai et al reported the issue of interobserver reproducibility of lesions in biopsy specimens of prostate glands including ASAP (29). The concordance rate of Gleason grading between general pathologists and urologic pathologists was only $47.5 \%$. Moreover, of the 23 biopsy specimens signed out as ASAP by general pathologists, 4 (17.4) and 4 (17.4\%) cancers were diagnosed as Gleason score 6 and 7, respectively, on the review of urologic pathologists. ASAP might have been underdiagnosed on initial biopsy in the present cohort, leading to the high incidence of Gleason grade greater than 6 on repeat biopsy after a diagnosis of ASAP.

Limitations of the present study include its retrospective and single-institutional nature and the small cohort, which potentially would cause a degree of bias. The criteria for the implementation of repeat biopsy, the methodology of biopsy, and the follow-up protocol were not consistent throughout the study period. Patients who received pre-biopsy magnetic resonance imaging followed by the addition of targeted biopsy cores might have a better chance to be diagnosed as having cancer, which would affect the rate of cancer detection. Nonetheless, the present study reflects clinical practice after a diagnosis of ASAP in a real-world setting, in which physicians' discretion and patients' preference play a crucial role in the decision-making process. Considering that no prospective study on ASAP has been available so far, well-defined prospective studies need to be conducted to establish the optimal management strategies for patients with a diagnosis of ASAP.

In conclusion, patients with repeat biopsy had higher PSA velocity and shorter PSA doubling time than patients without repeat biopsy after a diagnosis of ASAP on initial biopsy in a real-world setting. Small prostate volume and increasing age were identified as statistically significant predictors of cancer detection on repeat biopsy after a diagnosis of ASAP. Consideration of prostate volume and age would aid in the decision-making process to perform repeat biopsy in patients with high PSA velocity and short PSA doubling time after a diagnosis of ASAP.

\section{Acknowledgements}

The authors would like to thank Dr Kazumasa Oka (Department of Pathology, Hyogo Prefectural Nishinomiya Hospital) for the pathological evaluation of specimens.

\section{Funding}

No funding was received. 


\section{Availability of data and materials}

The datasets used and/or analyzed in the present study are available from the corresponding author on reasonable request.

\section{Authors' contributions}

TI, TY, and KN conceived and designed the current study. TI and TY performed the statistical analysis and wrote the manuscript. TI, TY, AT, KY and HK acquired, analyzed and interpreted the data, and wrote and critically revised the manuscript for important intellectual content. All authors read and approved the final manuscript.

\section{Ethics approval and consent to participate}

The present study was approved by the Research Ethics Board of the Hyogo Prefectural Nishinomiya Hospital (approval no. H30-37). Due to the retrospective nature of the study, informed consent was waived per protocol.

\section{Patient consent for publication}

Not applicable.

\section{Competing interests}

The authors declare that they have no competing interests.

\section{References}

1. Siegel RL, Miller KD and Jemal A: Cancer statistics, 2017. CA Cancer J Clin 67: 7-30, 2017.

2. Kimura T and Egawa S: Epidemiology of prostate cancer in Asian countries. Int J Urol 25: 524-531, 2018.

3. Saishin Gan Toukei (The Latest Cancer Statistics in Japan). https://ganjoho.jp/reg_stat/statistics/stat/summary.html. Accessed Jul 1, 2019.

4. Litwin MS and Tan HJ: The diagnosis and treatment of prostate cancer: A review. JAMA 317: 2532-2542, 2017.

5. Romero-Otero J, García-Gómez B, Duarte-Ojeda JM, RodríguezAntolín A, Vilaseca A, Carlsson SV and Touijer KA: Active surveillance for prostate cancer. Int J Urol 23: 211-218, 2016.

6. Montironi R, Scattoni V, Mazzucchelli R, Lopez-Beltran A, Bostwick DG and Montorsi F: Atypical foci suspicious but not diagnostic of malignancy in prostate needle biopsies (also referred to as 'atypical small acinar proliferation suspicious for but not diagnostic of malignancy'). Eur Urol 50: 666-674, 2006.

7. Epstein JI and Herawi M: Prostate needle biopsies containing prostatic intraepithelial neoplasia or atypical foci suspicious for carcinoma: Implications for patient care. J Urol 175: 820-834, 2006.

8. Scattoni V, Roscigno M, Freschi M, Dehò F, Raber M, Briganti A, Fantini G, Nava L, Montorsi F and Rigatti P: Atypical small acinar proliferation (ASAP) on extended prostatic biopsies: Predictive factors of cancer detection on repeat biopsies. Arch Ital Urol Androl 77: 31-36, 2005.

9. Borboroglu PG, Sur RL, Roberts JL and Amling CL: Repeat biopsy strategy in patients with atypical small acinar proliferation or high grade prostatic intraepithelial neoplasia on initial prostate needle biopsy. J Urol 166: 866-870, 2001.

10. Carroll PR, Parsons JK, Andriole G, Bahnson RR, Barocas DA Catalona WJ, Dahl DM, Davis JW, Epstein JI, Etzioni RB, et al: Prostate cancer early detection, version 1.2014. Featured updates to the NCCN guidelines. J Natl Compr Canc Netw 12: 1211-1219, 2014.

11. Heidenreich A, Bastian PJ, Bellmunt J, Bolla M, Joniau S, van der Kwast T, Mason M, Matveev V, Wiegel T, Zattoni F, et al: EAU guidelines on prostate cancer. Part 1: Screening, diagnosis, and local treatment with curative intent-update 2013. Eur Urol 65: 124-137, 2014.
12. Ynalvez LA, Kosarek CD, Kerr PS, Mahmoud AM,Eyzaguirre EJ, Orihuela E, Sonstein JN and Williams SB: Atypical small acinar proliferation at index prostate biopsy: Rethinking the re-biopsy paradigm. Int Urol Nephrol 50: 1-6, 2018.

13. Leone L, Lacetera V, Montironi R, Cantoro U, Conti A, Sbrollini G, Quaresima L, Mariani L, Muzzonigro G and Galosi AB: Biopsy follow-up in patients with isolated atypical small acinar proliferation (ASAP) in prostate biopsy. Arch Ital Urol Androl 86: 332-335, 2014.

14. Leone A, Rotker K, Butler C, Mega A, Li J, Amin A, Schiff SF, Pareek G, Golijanin D and Renzulli JF II: Atypical small acinar proliferation: Repeat biopsy and detection of high grade prostate cancer. Prostate Cancer 2015: 810159, 2015.

15. Leone A, Gershman B, Rotker K, Butler C, Fantasia J, Miller A, Afiadata A, Amin A, Zhou A, Jiang Z, et al: Atypical small acinar proliferation (ASAP): Is a repeat biopsy necessary ASAP? A multi-institutional review. Prostate Cancer Prostatic Dis 19: 68-71, 2016.

16. Warlick C, Feia K, Tomasini J, Iwamoto C, Lindgren B and Risk M: Rate of Gleason 7 or higher prostate cancer on repeat biopsy after a diagnosis of atypical small acinar proliferation. Prostate Cancer Prostatic Dis 18: 255-259, 2015.

17. Koca O, Calışkan S, Oztürk Mİ, Güneş M and Karaman MI: Significance of atypical small acinar proliferation and high-grade prostatic intraepithelial neoplasia in prostate biopsy. Korean $\mathrm{J}$ Urol 52: 736-740, 2011.

18. Ryu JH, Kim YB, Lee JK, Kim YJ and Jung TY: Predictive factors of prostate cancer at repeat biopsy in patients with an initial diagnosis of atypical small acinar proliferation of the prostate. Korean J Urol 51: 752-756, 2010.

19. Srirangam V, Rai BP, Abroaf A, Agarwal S, Tadtayev S, Foley C, Lane T, Adshead J and Vasdev N: Atypical small acinar proliferation and high grade prostatic intraepithelial neoplasia: Should we be concerned? An observational cohort study with a minimum follow-up of 3 years. Curr Urol 10: 199-205, 2017.

20. Cool DW, Romagnoli C, Izawa JI, Chin J, Gardi L, Tessier D, Mercado A, Mandel J, Ward AD and Fenster A: Comparison of prostate MRI-3D transrectal ultrasound fusion biopsy for first-time and repeat biopsy patients with previous atypical small acinar proliferation. Can Urol Assoc J 10: 342-348, 2016.

21. Aglamis E, Kocaarslan R, Yucetas U, Toktas G, Ceylan C, Doluoglu OG and Unluer E: How many cores should be taken in a repeat biopsy on patients in whom atypical small acinar proliferation has been identified in an initial transrectal prostate biopsy? Int Braz J Urol 40: 605-612, 2014.

22. Merrick GS, Galbreath RW, Bennett A, Butler WM and Amamovich E: Incidence, grade and distribution of prostate cancer following transperineal template-guided mapping biopsy in patients with atypical small acinar proliferation. World $\mathbf{J}$ Urol 35: 1009-1013, 2017.

23. Scattoni V, Roscigno M, Freschi M, Briganti A, Fantini GV, Bertini R, Salonia A, Montorsi F and Rigatti P: Predictors of prostate cancer after initial diagnosis of atypical small acinar proliferation at 10 to 12 core biopsies. Urology 66: 1043-1047, 2005.

24. Ficarra V, Novella G, Novara G, Galfano A,Pea M, Martignoni G and Artibani W: The potential impact of prostate volume in the planning of optimal number of cores in the systematic transperineal prostate biopsy. Eur Urol 48: 932-937, 2005.

25. Memorial Sloan Kettering Cancer Center, PSA Doubling Time. https://www.mskcc.org/nomograms/prostate/psa_doubling_time. Accessed Aug 1, 2019.

26. Paller CJ, Olatoye D, Xie S, Zhou X, Denmeade SR, Eisenberger MA, Antonarakis ES, Carducci MA and Rosner GL: The effect of the frequency and duration of PSA measurement on PSA doubling time calculations in men with biochemically recurrent prostate cancer. Prostate Cancer Prostatic Dis 17: 28-33, 2014

27. Letran JL, Meyer GE, Loberiza FR and Brawer MK: The effect of prostate volume on the yield of needle biopsy. J Urol 160: 1718-1721, 1998.

28. Leibovici D, Shilo Y, Raz O, Stav K, Sandbank J, Segal M and Zisman A: Is the diagnostic yield of prostate needle biopsies affected by prostate volume? Urol Oncol 31: 1003-1005, 2013.

29. Nakai Y, Tanaka N, Shimada K, Konishi N, Miyake M, Anai S and Fujimoto K: Review by urological pathologists improves the accuracy of Gleason grading by general pathologists. BMC Urol 15: 70, 2015. 